\title{
A system for the assessment of human performance in concurrent verbal and manual control tasks
}

\author{
STEVEN D. HARRIS, ROBERT A. NORTH, and JERRY M. OWENS \\ U.S. Naval Aerospace Medical Research Laboratory, Pensacola, Florida 32508
}

\begin{abstract}
A multipurpose laboratory system incorporating a voice interactive terminal and graphic display system is described. The facility is designed to investigate some aspects of human performance on concurrent verbal and tracking tasks. The results of a pilot study showing decrements in dual-task performance are reported.
\end{abstract}

Military aviation systems are notorious for overloading their operators with information to be monitored, categorized, acted upon, and remembered. The myriad tasks required to accomplish a complex mission are usually performed simultaneously or in rapid succession under severe time constraints.

Several researchers have investigated the problems posed by the requirement placed on the human operator to maintain continuous control over a complex system while simultaneously receiving or transmitting verbal communications. To summarize the literature, it is clear that certain verbal tasks interfere with simultaneous performance of some tracking tasks. This interference may be due to response requirements of the verbal task. How ever, other characteristics, such as stimulus encoding requirements and memory load, are probably important. Moreover, it is certain that some parameters of the tracking task contribute to decrements in performance during simultaneous verbal information processing. (For a review of this area, see Harris, Note 1). A systematic investigation of these variables is needed in order to determine the extent of human operator performance capabilities in simultaneous verbal and manual control tasks. The Naval Aerospace Medical Research Laboratory (NAMRL) has developed a unique facility and program of research to investigate these questions.

Criteria for the design of the Laboratory's facility were relatively simple. The system had to be capable of: (1) presenting concurrent independent tasks in visual and auditory modes, (2) detecting and analyzing in real time subject responses in manual and oral modes, and (3) modifying the difficulty level of tasks in real time as a function of experimental variable manipulations or subject performance. Further, to avoid a long delay

The authors wish to acknowledge the contribution made by CDR R.S. Gibson to the design of the system reported in this paper. The computer programs were written by Mr. Greg Thorsen, Mr. Peter Collyer, and L. T. Harris at NAMRL. The opinions expressed in this paper are those of the authors, and should not be construed to represent policies of the Navy Department. before starting the research program, it was desirable that the system represent the state of technology, requiring no development of new hardware and minimum software development. The purpose of this paper is to describe the laboratory system and to present the results of a preliminary study designed to investigate the interaction of continuous tracking and a verbal information-processing task.

\section{EXPERIMENT CONTROLLER SYSTEMS}

The primary components of the laboratory facility are illustrated in Figures 1 and 2. The computer equipment consists of a Data General Corporation (DGC) NOVA 800 minicomputer with $32 \mathrm{~K}$ by 16 core memory that serves as the experiment controller, scheduling all task stimulus events, collecting responses, and performing the on-line analysis necessary for adapting the difficulty of experimental tasks in real time to individual subject skill levels, and providing feedback to subjects concerning performance. Peripheral to the NOVA 800

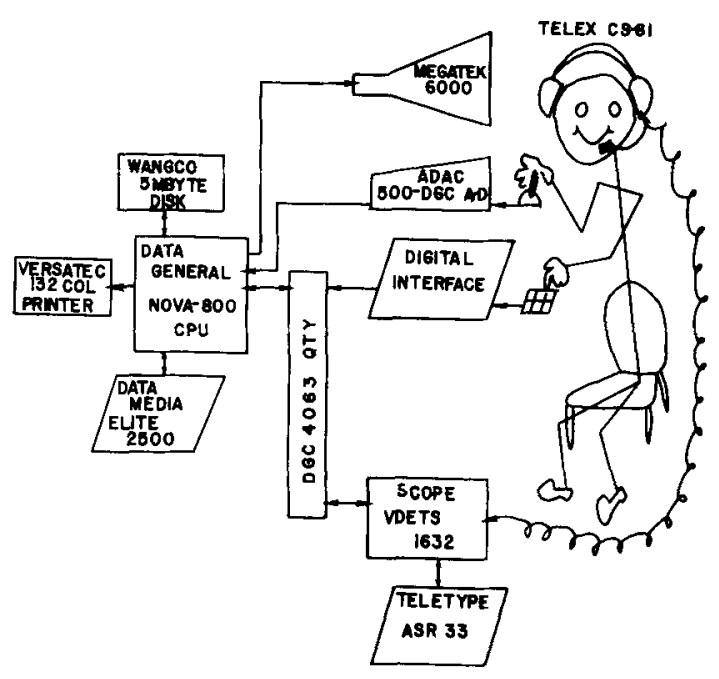

Figure 1. Experiment controller systems. 


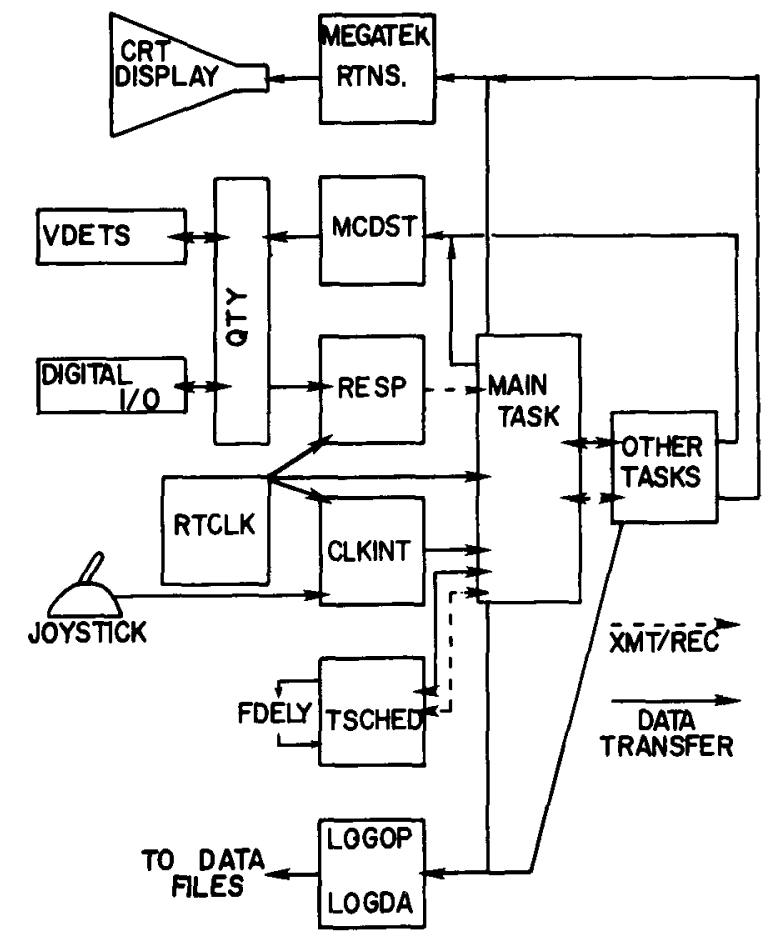

Figure 2. Experiment controller programs.

are a serial asynchronous data multiplexor with four duplex channels (a DGC QTY device); a 5-megabyte disk; a system console, a 132-column nonimpact line printer; an eight-channel analog-to-digital converter; a Megatek Series 6000 graphic system with a HewlettPackard Model 1310A CRT display and hardware character and vector generators; a Measurement Systems, Inc., Model 526 X-Y joystick; and a Scope Electronics Model 1632 Voice Data Entry Terminal System (VDETS), comprising a DGC NOVA $2 / 10 \mathrm{CPU}$ with $16 \mathrm{~K}$ by 16 core, a Federal Screw Works VOTRAX voice synthesizer, Model VS-6, and the Scope VDETS user station, Model VIP-100, and a Teletype console). ${ }^{1}$

The digital interface illustrated in Figure 1 was custom-built for the U.S. Navy, but serves a general function of detecting contact switch closures, encoding the switch number into an 8-bit representation, and generating an interrupt over one channel of the asynchronous multiplexor.

Figure 2 illustrates the organization of the experiment controlling programs. The blocks in Figure 2 labeled MCDST, RESP, RTCLK, CLKINT, TSCHED, and LOGOP LOGDA represent DGC real-time FORTRAN tasks or FORTRAN-callable assembly language subroutines, referred to collectively as the MARTSPKG (Multipurpose Automated Research Test Station Package). Communication among the software components of the MARTSPKG is accomplished via XMT-REC or subroutine calls for synchronization and transfer of data.

For example, when a contact switch closure is de- tected by the digital interface, an interrupt is generated. Task RESP, which has the highest priority of all components, gains control, reads the real-time clock, and places the input switch code data and a time tag into an array labeled COMMON. RESP then executes an XMT call on a key selected from a table relating switch codes to specific experimental tasks. When the appropriate task eventually gains control and executes a REC call, the data is available in COMMON with a time tag very close to the real time of response occurrence.

Generation of stimulus events is accomplished via subroutine call to MCDST for output over the QTY or to Megatek-supplied subroutines resident in the NOVA 800 for output to the CRT.

Communication with the VDETS terminal is accomplished in the same routines that perform discrete $I / O$ functions over the QTY. Voice synthesis and recognition functions are accomplished in the VDETS NOVA 2/10, using the VOICE operating system supplied by Scope Electronics. Synthesized voice vocabulary size is virtually unlimited. Size of the recognition vocabulary is limited to about 75 words. Current experiments utilize a vocabulary of about nine words for both synthesis and recognition. Word recognition accuracy for our subject population after 10 training passes through the vocabulary was above $99 \%$, with very low rejection rate. Mean latency of recognition is estimated at about $.8 \mathrm{sec}$, including utterance duration. Recognition latency is a function of vocabulary size, syntax structure, and length of utterance.

In summary, all of the major hardware components in the system are available from commercial manufacturers. Software consists of routines supplied by the manufacturers or custom programming developed by the U.S. Navy and available without charge, consistent with the civilian technology transfer policies of the Department of Defense.

The remainder of this paper describes a pilot experiment to investigate timesharing performance of human subjects in simultaneous verbal and tracking tasks.

\section{METHOD}

\section{Subjects}

Eight subjects were selected from the Naval Aerospace Medical Research Laboratory professional staff and from a group of Naval Flight Officer students. All subjects were male, right-handed, and between the ages of 22 and 35 years.

\section{Experimental Trial Sequence}

The experiment consisted of the single- and dual-task performance trials presented in Table 1. The order of Trials 1T1, $1 \mathrm{~T} 2,1 \mathrm{~V}$, and $1 \mathrm{~K}$ was counterbalanced across the eight subjects, as were Trials 2TV and 2TK. One minute of rest intervened between Trials $1 \mathrm{~T} 1$ and $1 \mathrm{~T} 2$ and between $2 \mathrm{~V}$ and $2 \mathrm{~K}$.

\section{Apparatus}

Subjects performed the tasks in a sound-attenuated booth equipped with joystick control for right-hand tracking and an eight-position soft-touch keyboard for the continous digit 
Table 1

Experimental Trial Sequence

\begin{tabular}{ll}
\hline Trial & \multicolumn{1}{c}{ Description } \\
\hline 1T1 & Single-Task Tracking (3 min) \\
1T2 & Single-Task Tracking (3 min) \\
1V & Single-Task Subtraction, Vocal Responses (50 trials) \\
1K & Single-Task Subtraction, Keyboard Response (50 trials) \\
2V & Dual-Task with Vocal Responses (3 min) \\
2K & Dual-Task with Keyboard Responses (3 min) \\
\hline
\end{tabular}

subtraction task. The keyboard task was performed with the left hand. A head-mounted noise-cancelling microphone was provided for the voice response condition of the continuous subtraction task. Single- and dual-task displays for tracking and continuous on-line feedback were presented on the HP 1310A CRT mounted in front of the subject. The display is illustrated in Figure 3. Ambient lighting and seat positioning were adjusted to provide comfortable viewing conditions for each subject.

\section{Single-Task Tracking}

One experimental task was continuous compensatory tracking, similar to that reported by North and Gopher (1976). The tracking task required the subject to keep a moving cursor centered on a vertical line within a horizontal track. Lateral control movements of the joystick moved the cursor left or right, and the subject was instructed to respond as quickly and accurately as possible in correcting for the random deviations of the cursor.

The forcing function input consisted of three nonharmonically related sinusoidal waveforms added to joystick position. For the first $4 \mathrm{~min}$ of single-task tracking, the difficulty of the task was adjusted through adaptive lobic. When the subject was inside $20 \%$ of scale error, the task increased in difficulty by increasing the ratio of acceleration to rate control dynamics. When outside this tolerance level, the task decreased in difficulty by decreasing this ratio. Initially, the controller acted as a pure rate control device. The adaptive process was relatively continuous, with adjustments every $50 \mathrm{msec}$ incrementing or decrementing acceleration percentage in steps of $.05 \%$. This adaptive manipulation of tracking difficulty brings each subject to his own individual difficulty level in a short time period, while keeping single-task error level relatively constant across subjects.

Acceleration control percentage was fixed for the final $2 \mathrm{~min}$ of the second single-task tracking period, during which root mean square error (RMSE) was averaged to represent the subject's single-task tracking performance.

On-line performance feedback was presented to the subject during tracking performance by means of a continuously updating bar graph. The bar increased and decreased in height with

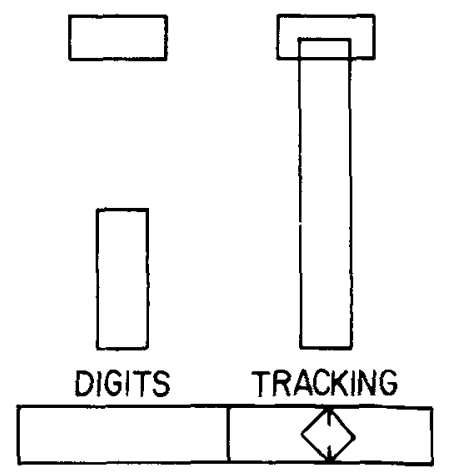

Figure 3. CRT display. improving or declining performance, respectively. A desired level of performance, indicated by a small rectangular box, represented the $20 \%$ of scale tolerance level used to manipulate the adaptive variable. Subjects were instructed to reach and maintain this level or better during the trial. In single-task performance the feedback display was based on time on target ( $20 \%$ error) averaged over the last $10 \mathrm{sec}$ and updated twice each second.

\section{Continuous Digit Subtraction}

The digit task required the subject to compute the absolute difference between the immediately preceding and currently presented numbers in a random sequence of digits ranging from 0 to 7. Digit presentation was auditory (VOTRAX system) for the two response conditions of the task. The first response condition was vocalization of the absolute difference; the second required keyboard responses. The subject keyed or vocalized the initial digit to begin the task, after which he was required to remember the preceding digit to compute the absolute difference. The task was subject paced; that is, a new digit was immediately presented when the subject responded correctly. Single-task performance consisted of 50 trials in each condition.

Prior to requiring voice responses, the subject programmed the VDETS terminal by sequentially speaking the nine response alternatives a total of 10 times. A calibration of the system's recognition capability was then performed by the experimenter noting the recognition scores on a subsequent test of the eight numbers, and the VDETS recognition threshold was adjusted accordingly to provide maximum recognition probability during an experimental trial. Digits with low recognition scores were reprogrammed by the subject prior to the experimental trial.

In the event the subject forgot the previous digit, he could recall the item by saying the word "again" (an additional item programmed during VDETS calibration by each subject) or by pressing the " 8 " key in the keyboard response condition. This action resulted in a repeat of both the previous and the current digits. In the event that the VDETS system failed to recognize the subject's utterance, VOTRAX responded with the phrase "say again", asking the subject to repeat his answer.

On-line feedback for the digit subtraction task was also provided. The vertical bar graph on the left side of the CRT display (see Figure 3) represented the time the subject took to complete each trial. The desired level of performance was initially set at $3.0 \mathrm{sec}$ but was adjusted to represent the subject's current best performance over 10 successive trials if this value became less than $3.0 \mathrm{sec}$. Thus, the feedback and desired performance goal served to drive the subject to his maximum output on the digit task.

\section{Dual-Task Performance Trials}

Two dual-task trials, one with keyboard digit task response requirement and one with vocal response requirement, followed single-task performance. Subjects were instructed that the tasks were of equal priority and told to allocate approximately equal attention to them. On-line momentary feedback for each task was presented (see Figure 3), and the desired performance goals represented mean performance during the appropriate single-task trials. Performance measures were identical to that of single-task performance for both tasks. The momentary height of the tracking feedback graph was computed by subtracting the current error score from the single-task mean RMS score and dividing by the standard deviation of single-task performance. Thus, the bar moved within a standardized score space for each subject. An analogous computation was made for the time required to complete each trial on the digit task.

\section{RESULTS}

Only data from experimental sessions on Day 2 were analyzed. The experiment was organized as a two- 
factor subjects by treatments design. The two independent variables were processing load (Conditions ST, single-task performance, and DT, dual-task performance) and digit task response mode (Conditions DK, digit task keyboard mode, and DV, voice mode). Three categories of errors were possible in the digit-processing task: incorrect responses made by the subject, misrecognition of a correct response by the VDETS terminal, and failure of the VDETS algorithms to recognize a response at all (called node failures). Because errors in the data can be introduced by the system, caution is in order when interpreting the results. It was decided to examine first only trials in which no error of any kind occurred. The effects of processing load and response mode on latency of correct responses are summarized in Figure 4. Analysis of variance indicated that only response mode reliably affected latency $(F=41.764, \mathrm{df}=1 / 6, \mathrm{p}<.005)$. Because of the inherent lag in the recognition of voiced responses by the system, an estimate of recognition latency was derived to subtract from the response time data. This estimate was obtained by training the recognition component of the VDETS terminal to recognize synthesized speech generated by the VOTRAX component. These data are summarized in Table 2 . When the mean recognition time for synthesized speech $(.527 \mathrm{sec})$ was subtracted from the subject response data, the result suggested that the difference in response time apparent in Figure 4 was due to hardware limitations, not to limits in the subjects' information-processing capacity in the voice mode.

Analysis of variance was also performed on data from trials containing errors of two kinds: incorrect responses and requests for the stimulus to be repeated. Trials containing node failures were again excluded from the analysis. Data from five subjects were analyzed. The remaining three subjects were deleted due to lost data or because they failed to generate errors or to request repeated stimuli.

Error density was computed as the sum of errors and repeats divided by the total number of correct responses in the session. The results of analyses of variance indicated a reliable effect of the processing

Table 2

Mean Recognition Latencies and Utterance Durations in Seconds

\begin{tabular}{ccc}
\hline Stimulus & $\begin{array}{c}\text { Recognition } \\
\text { Latency }\end{array}$ & $\begin{array}{c}\text { Utterance } \\
\text { Duration }\end{array}$ \\
\hline 0 & .493 & .325 \\
1 & .473 & .250 \\
2 & .473 & .250 \\
3 & .552 & .300 \\
4 & .535 & .275 \\
5 & .624 & .300 \\
6 & .518 & .375 \\
7 & .544 & .250 \\
Mean & .527 & .291 \\
\hline
\end{tabular}

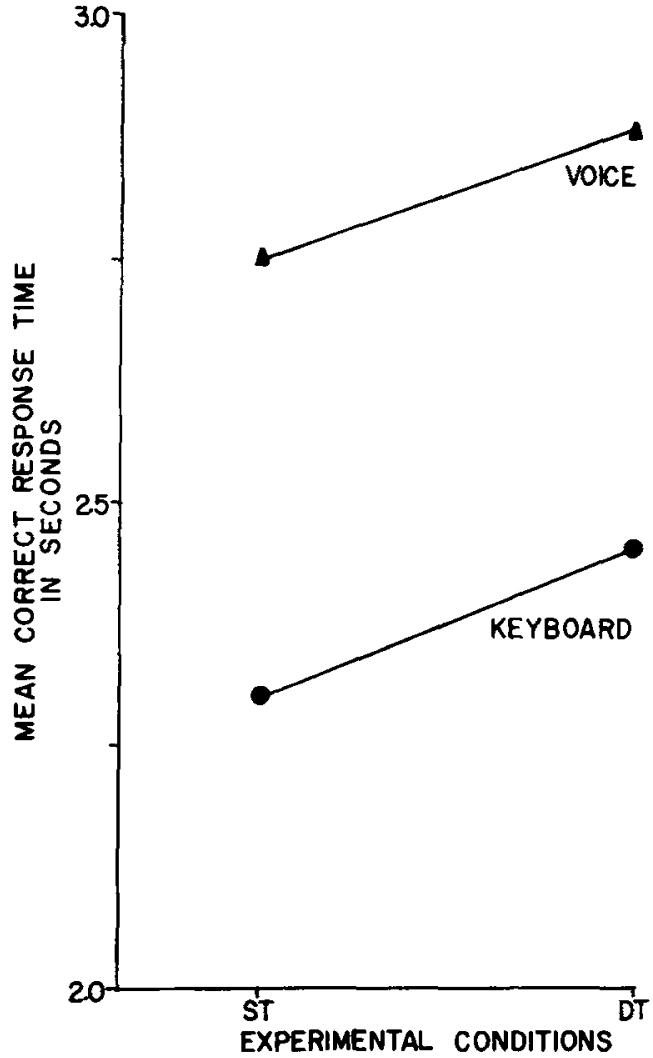

Figure 4. Mean correct response time as a function of processing load and response mode on the digit task.

load variable $(F=19.968, \mathrm{df}=1 / 4, \mathrm{p}<.05)$. Figure 5 presents these results in graphic form.

Finally, RMS tracking error data from all eight subjects were analyzed comparing the effects of three conditions: single-task tracking, dual-task Condition DK, and dual-task Condition DV. This analysis showed a reliable main effect of conditions on performance $(\mathrm{F}=35.25, \mathrm{df}=1 / 7, \mathrm{p}<.001)$. The results are illustrated in Figure 6. A subsequent Newman-Keuls test between pairwise differences of condition means revealed a difference between Conditions ST and DK $(\mathrm{p}<.01)$, ST and DV $(\mathrm{p}<.05)$, and DK and DV $(\mathrm{p}<.01)$. (Standard error within conditions $=.0207$, $\mathrm{df}=7$.)

\section{DISCUSSION}

Concurrent tracking appears to have a reliable effect on one of the dependent measures of digit-processing performance that was analyzed. The effect of processing load on the error density data approached an acceptable level of statistical reliability and should be examined in future studies. The results suggest that concurrent tracking does not delay processing of correct responses, but may cause occasional distraction, resulting in errors or a request for the stimulus to be repeated. Of course, this must be viewed as a tentative conclusion, since two 


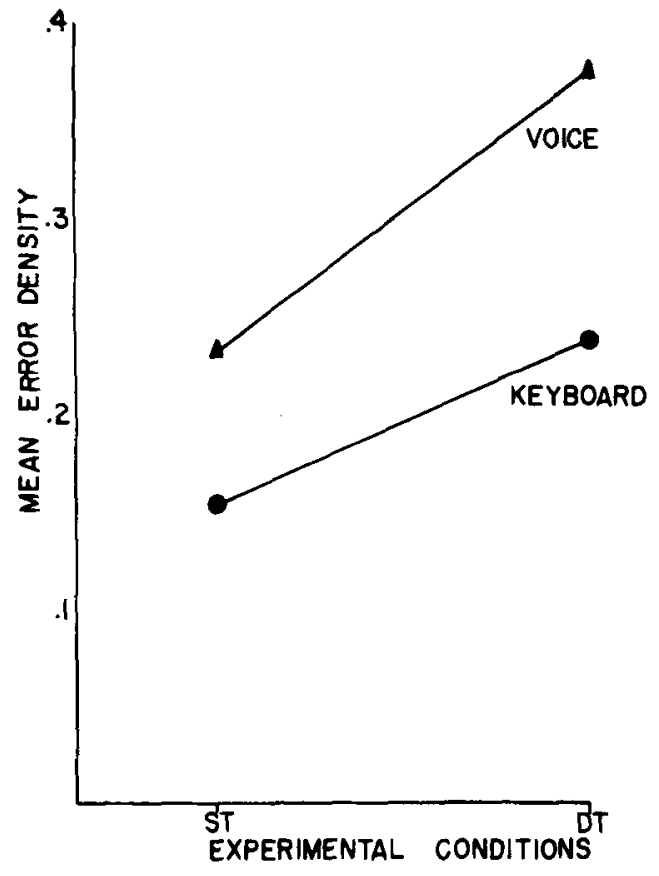

Figure 5. Mean error density as a function of processing load and response mode on the digit task.

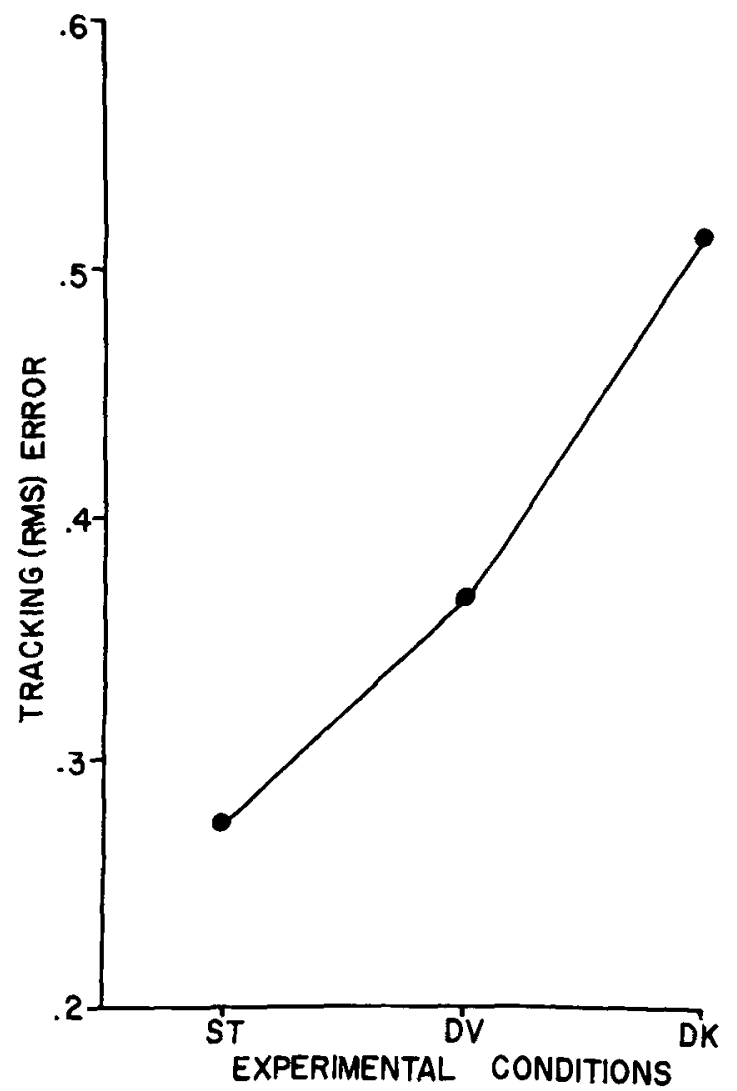

Figure 6. RMS tracking error as a function of processing load and response mode on the digit task.

of the eight subjects failed to make errors on the digit task and were excluded from the analysis.
The effect of concurrent digit processing on tracking performance is much clearer. The results indicate that the voice response mode caused less decrement in tracking performance than did the keyboard mode, even though subjects had considerable practice with the keyboard and it contained only nine keys, arranged to provide high S-R compatibility. The fact that any decrement occurred is surprising, since both conditions employed independent stimulus and response modes for the two concurrent tasks. It is possible that the differential effects of voice and keyboard response modes on tracking performance are due to assignment of different priorities to the two tasks by the subjects. The attempt to control priorities using visual feedback may have been unsuccessful. However, no reliable difference between performance measures on the digit task in the two response modes was observed in the data. If subjects were able to maintain the desired priorities, then the differential effects of voice and keyboard modes on tracking performance argue against adopting a simple, single-channel model of the operator to explain the results.

In two recent papers, Norman and Bobrow (1975, 1976) proposed the concept of processing resources to describe the limited facilities available to an information-processing system for executing its programs. When two processes require access to the same resource, that resource must be allocated between them. Performance on one or both of the competing processes deteriorates when the amount of the resource required by both processes exceeds the limit available to the system. Their model implies that interference between complex tasks performed concurrently is due to competition for specialized resources, and the analysis of the interference requires examination of the specific processing requirements of the tasks. Experiments now under way at NAMRL are designed to address this problem by examining the performance operating characteristics of the tasks reported in this paper.

\section{REFERENCE NOTE}

1. Harris, S. D. Human performance in concurrent verbal and tracking tasks: $A$ review of the literature. Pensacola, Fla: Naval Aerospace Medical Research Laboratory, 1977, in press.

\section{REFERENCES}

Norman, D., \& Bobrow, D. G. On data-limited and resourcelimited processes. Cognitive Psychology, 1975, 7, 44-64.

Noruman, D. A., \& Bobrow, D. G. On the analysis of performance operating characteristics. Psychological Review, 1976, 83, 508-510.

Norte, R. A., \& GoPHER, D. Measures of attention as predictors of flight performance. Human Factors, 1976, 18, 1-14.

\section{NOTE}

1. Scope Electronics sold its interest in voice interactive terminals to Interstate Electronics of California after this paper was prepared. 